\title{
DIRECT ESTIMATES IN SIMULTANEOUS APPROXIMATION FOR DURRMEYER TYPE OPERATORS
}

\author{
N. K. GOVIL AND ViJAY GUPTA
}

\begin{abstract}
In the present paper, we study a Durrmeyer type integral modification of the wellknown Baskakov operators with the weight function of Beta basis function. Some approximation properties of these operators were recently studied by Finta [2]. Here we study simultaneous approximation properties for these operators. We estimate local direct result in terms of modulus of continuity. The operators considered in this paper reproduce not only the constant functions but also the linear ones, due to this property we can improve the order of approximation for these operators by applying the iterative combinations, which were first studied by Micchelli [7]. We establish an asymptotic formula and error estimation in terms of higher order modulus of continuity in simultaneous approximation for the Micchelli combinations of these operators.
\end{abstract}

Mathematics subject classification (2000): 41A30, 41A36.

Key words and phrases: simultaneous approximation, Micchelli combinations, asymptotic formula, error estimate.

\section{REFERENCES}

[1] P. N. AgRaWAL, A. J. MoHAmmad, Approximation by iterative combinations of a new sequence of linear positive operators, Advances in Constructive Approximation, Vanderbilt 2003, 13-24, Mod Methods Math., Nashboro Press, Brentwood, TN, 2004.

[2] Z. FinTA, On converse approximation theorems, J. Mathematical Analysis and Applications, 312, (1) (2005), 159-180.

[3] S. Goldberg, V. MeIR, Minimum moduli of ordinary differential operators, Proc. London Math. Soc., 23, (1971), 1-15.

[4] V. GuPTA, A note on modified Baskakov type operators, Approx. Theory and its Appl., 10, (3) (1994), 74-78.

[5] V. GUPTA, Error estimation by mixed summation integral type operators, J. Mathematical Analysis and Applications, 313, (2) (2006), 632-641.

[6] M. K. GUPTA, V. VASISHTHA, The iterative combinations of a new sequence of linear positive operators, Math Comput Modelling, 39, (2004), 521-527.

[7] C. A. Micchelli, Saturation class and iterates of the Berntein polynomials, J. Approx. Theory, 8, (1973), 1-18.

[8] H. M. SRIVASTAVA, V. GUPTA, A certain family of summation integral type operators, Math and Comput. Modelling, 37, (2003), 1307-1315.

[9] Y. WANG, S. GuO, Rate of approximation of functions of bounded variation by modified Lupas operators, Bull. Austral. Math Soc., 44, (1991), 177-188. 